\title{
RANDOM OPTIMIZATION ON RANDOM SETS
}

\author{
EMMANUEL LEPINETTE
}

\begin{abstract}
Random sets and random preorders naturally appear in financial market modelling with transaction costs. In this paper, we introduce and study a concept of essential minimum of a family of vector-valued random variables, i.e. the set of all minimal elements with respect to some random preorder. We provide some conditions under which the essential minimum is not empty and we present two applications in optimisation to Mathematical Finance and Economics.
\end{abstract}

\section{INTRODUCTION}

Random sets and random preorders naturally appear in financial market modelling with transaction costs. Indeed, a fundamental concept to model a financial market with transaction costs is the random solvency set $\mathbf{G}_{t}$ of all financial portfolio positions which is possible to liquidate without any debt at time $t$. Since the pioneering paper [9], models with proportional transaction costs have been extended to the case where the solvency sets are random convex cones of $\mathbb{R}^{d}, d \geqslant 1$, see [13, Section 3]. They are now very popular in Mathematical Finance. Actually, it is possible to consider very general models, e.g. models with convex or fixed costs, through the concept of liquidation value process $\left(\mathrm{E}_{t}\right)_{t \geqslant 0}$ so that $\mathbf{G}_{t}=\left\{z \in \mathbb{R}^{d}: \mathrm{E}_{t}(z) \geqslant 0\right\}$ is the set of all financial positions whose liquidation values are non negative at time $t$. Notice that $\mathbf{G}_{t}$ is random as it depends on the future prices observed in the financial market at time $t$. Associated to these random solvency sets, the random preorders defined by $x \gg_{t} y$ when $x-y \in \mathbf{G}_{t}$ naturally appear as a portfolio process $\left(V_{t}\right)_{t=0,1, \ldots}$ satisfies by definition the dynamics $V_{t}-V_{t-1} \in-\mathbf{G}_{t}$ in discrete time, see [13, Section 3.1.1], i.e. $V_{t-1} \gg_{t} V_{t}, t \geqslant 1$. This leads to consider a new approach based on random preorders and optimization on random sets when solving the classical problem of super-replication, i.e. for a given measurable

Date: May 14, 2018.

2010 Mathematics Subject Classification. 91G20, 60D05, 60G42.

Key words and phrases. Random preference relations, Random sets, Super hedging, Transaction costs. 
payoff $\xi$ at time $T$, characterising the set of all portfolio processes $\left(V_{t}\right)_{t=0,1, \cdots, T}$ which satisfy $V_{T} \gg_{T} \xi$. This approach seems to be fruitful for non conical models and, more generally for non convex models, see [15] with fixed costs. Indeed, classical tools from convex analysis are no more appropriate. Precisely, it is not possible to characterise the super-hedging prices through the duals elements, i.e. the risk-neutral probability measures for frictionless models, see [3], and the Consistent Price Systems, i.e. martingales evolving in the positive dual of the solvency sets, for models with proportional transaction costs, see $[13$, Section 3.3]. Notice that a set-valued optimisation approach has been successfully applied to compute the super-hedging prices of European payoffs in [2] when the probability space is finite. The same approach is also used in the setting of set-valued risk measures in presence of proportional transaction costs, $[7,6]$, where the random orders defined by the solvency sets play a crucial role. More recently, a conditional analysis approach is considered to solve stochastic optimal problems in discrete-time [8] with applications in Finance and Economics. Similarly, a backward approach is implemented in [1]. These works are based on new ideas stemming from conditional analysis applied to the theory of random sets and measurable selections.

For non convex models, we also need to take a fresh look. A new idea is to consider only the portfolio processes which are minimal in some sense to be defined with respect to the random preorders. This idea has been initially introduced, see [10, 11, 12], for conic models and the concept of essential supremum for a family of vector-valued random variables is formulated with respect to the random preorder defined by a convex cone. In the present paper, we consider a more general setting where the random preorder is either defined by a random set which is not necessarily convex or is defined by a random countable multi-utility representation. We introduce the concept of essential minimum for a family of vector-valued random variables with respect to a random preorder. Our main contribution is to show the existence of minimal elements, i.e. the essential minimum is not empty, under mild conditions. Finally, we illustrate our result by two applications. The first one in Mathematical Finance improves a result of [15] by characterizing the minimal portfolio processes super-replicating a European claim. The second one is a classical problem in Economics which is here solved in a random environment, i.e. we minimise a random cost function on a random set.

In the following, we first recall the notions of random set (see [16] for a complete overview) and random preorders with examples. The 
main result (Theorem 4.1) is then formulated. At last, we present the two applications.

\section{Measurability of RANDOM SETS}

Let $\mathbb{R}^{d}$ be the Euclidean space with norm $\|\cdot\|$ and the Borel $\sigma$-algebra $\mathcal{B}\left(\mathbb{R}^{d}\right)$. The closure of a set $A \subset \mathbb{R}^{d}$ is denoted by $\operatorname{cl} A$.

A set-valued mapping $\omega \mapsto X(\omega) \subset \mathbb{R}^{d}$ from a complete probability space $(\Omega, \mathcal{F}, \mathrm{P})$ to the family of all subsets of $\mathbb{R}^{d}$ is called $\mathcal{F}$-measurable if its graph

$$
\operatorname{Gr} X=\left\{(\omega, x) \in \Omega \times \mathbb{R}^{d}: x \in X(\omega)\right\} \subset \Omega \times \mathbb{R}^{d}
$$

belongs to the product $\sigma$-algebra $\mathcal{F} \otimes \mathcal{B}\left(\mathbb{R}^{d}\right)$. In this case, $X$ is said to be a random set. In the same way, the $\mathcal{H}$-measurability of $X$ with respect to a sub- $\sigma$-algebra $\mathcal{H}$ of $\mathcal{F}$ is defined. Unless otherwise stated, by the measurability we always understand the measurability with respect to $\mathcal{F}$. The random set $X$ is said to be closed if $X(\omega)$ is a closed set for almost all $\omega$.

Definition 2.1. An $\mathcal{F}$-measurable random element $\xi$ in $\mathbb{R}^{d}$ such that $\xi(\omega) \in X(\omega)$ for almost all $\omega \in \Omega$ is said to be an $\mathcal{F}$-measurable selection (selection in short) of $X, \mathcal{L}^{0}(X, \mathcal{F})$ denotes the family of all $\mathcal{F}$ measurable selections of $X$, and $\mathcal{L}^{p}(X, \mathcal{F})$ is the family of $p$-integrable ones.

By [4, Th. 4.4], an a.s. non-empty random set $X$ has at least one selection, i.e. $\mathcal{L}^{0}(X, \mathcal{F}) \neq \varnothing$ where $\mathcal{L}^{0}\left(\mathbb{R}^{d}, \mathcal{F}\right)$ is equipped with the topology generated by the convergence in probability. When such a property is used to mention the existence of such a measurable selection, we write by measurable selection argument.

Definition 2.2. A family $\Xi \subset \mathcal{L}^{0}\left(\mathbb{R}^{d}, \mathcal{F}\right)$ is said to be $\mathcal{H}$-decomposable if

$$
\sum_{n=1}^{m} \xi_{n} \mathbf{1}_{A_{n}} \in \Xi
$$

for all finite sequences $\left(\xi_{n}\right)_{n=1, \cdots, m}$ from $\Xi$ and all finite $\mathcal{H}$-measurable partitions $\left(A_{n}\right)_{n \geqslant 1}$ of $\Omega$.

The following result is well known for $p=1[5]$, for $p \in[1, \infty][16$, Th. 2.1.6], and is proven in [14] for $p=0$.

Theorem 2.3. Let $\Xi$ be a non-empty subset of $\mathcal{L}^{p}\left(\mathbb{R}^{d}, \mathcal{F}\right)$ for $p=0$ or $p \in[1, \infty]$. Then

$$
\Xi \cap \mathcal{L}^{p}\left(\mathbb{R}^{d}, \mathcal{H}\right)=\mathcal{L}^{p}(X, \mathcal{H})
$$


for an $\mathcal{H}$-measurable random closed set $X$ if and only if $\Xi$ is $\mathcal{H}$ decomposable and closed.

We deduce the following result [14]. In particular, the existence of a countable respresentation of a random set is well known when it is closed, see e.g. [16, Th. 2.2.3]. It is called the Castaing representation.

Proposition 2.4. If $X$ is a random set, then its pointwise closure cl $X(\omega), \omega \in \Omega$, is a random closed set, and $\mathcal{L}^{0}(\operatorname{cl} X, \mathcal{F})=\operatorname{cl}_{0} \mathcal{L}^{0}(X, \mathcal{F})$. Furthermore, there exists a countable family $\left(\xi_{i}\right)_{i \geqslant 1}$ of measurable selections of $X$ such that $\operatorname{cl} X=\operatorname{cl}\left\{\xi_{i}, i \geqslant 1\right\}$ a.s.

\section{RANDOM PREORDERS}

In the following, we consider a random binary relation (or preference relation) between the elements of $\mathbb{R}^{d}$ denoted by 》 which is reflexive. In the case where $\gg$ is also transitive, $\gg$ is a random preorder. Note that it is naturally possible to extend it to the set of all measurable random variables, i.e. for any $\gamma_{1}, \gamma_{2} \in L^{0}\left(\mathbb{R}^{d}, \mathcal{F}\right)$, we write $\gamma_{1} \gg \gamma_{2}$ when the set of all $\omega$ such that $\gamma_{1}(\omega) \gg \gamma_{2}(\omega)$ is of full measure. We shall consider on a complete probability space $(\Omega, \mathcal{F}, \mathrm{P})$ two types of random preorders we define below. To do so, we need to introduce the following notions.

Lemma 3.1. Let $\mathcal{H}$ be a sub $\sigma$-algebra of $\mathcal{F}$ and let $h: \Omega \times \mathbb{R}^{k} \rightarrow \mathbb{R}$. Then $h$ is $\mathcal{H}$-normal integrand (see Definition 14.27 in [18]) if and only if $h$ is $\mathcal{H} \otimes \mathcal{B}\left(\mathbb{R}^{k}\right)$-measurable and is lower semi-continuous (l.s.c. in the sequel) in $x$, see [18, Corollary 14.34].

Consider $Z \in L^{0}\left(\mathbb{R}^{k}, \mathcal{H}\right)$. We shall use the notation $h(Z): \omega \rightarrow$ $h(Z(\omega))=h(\omega, Z(\omega))$. If $h$ is $\mathcal{H} \otimes \mathcal{B}\left(\mathbb{R}^{k}\right)$-measurable, $h(Z) \in$ $L^{0}\left(\mathbb{R}^{k}, \mathcal{H}\right)$.

Definition 3.2. We say that the random preorder is of type I if there exists a super additive ${ }^{1}$ random function $L$ with $L(0)=0$ such that for all $x, y \in \mathbb{R}^{d}, x \gg y$ if and only if $L(x-y) \geqslant 0$ a.s. Moreover, we suppose that $-L$ is a $\mathcal{F}$-normal integrand and there exists a bounded $\mathcal{F}$-normal integrand $u$ such that for all $x, y \in \mathbb{R}^{d}, L(x-y)=0$ implies that $u(x) \geqslant u(y)$ with a strict inequality if $x \neq y$.

Definition 3.3. We say that the random preference relation is of type II if there exists a countable family of random functions $\left(u_{i}\right)_{i \in \mathbb{N}}$ which are $\mathcal{F}$-normal integrands such that for all $x, y \in \mathbb{R}^{d}, x \gg y$ if and only if $u_{i}(x) \geqslant u_{i}(y)$ a.s. for all $i \in \mathbb{N}$.

\footnotetext{
1.e. $L(x+y) \geqslant L(x)+L(y)$.
} 
Example 3.4. Let $G$ be a random $\mathcal{F}$-measurable closed set of $\mathbb{R}^{d}, d \geqslant 1$, containing $\mathbb{R}_{+} e_{1}$ where $e_{1}=(1,0, \cdots, 0) \in \mathbb{R}^{d}$. Assume that $G+G \subset$ $G$ a.s. and $G \cap(-G)=\{0\}$. Moreover, suppose that the smallest closed convex cone $K=\operatorname{cl}\left(\mathbb{R}_{+} G\right)$ containing $G$ does not contain $-e_{1}$. In mathematical finance, $G$ is interpreted as the set of all financial positions which are solvent, i.e. that may be liquidated without any debt. Let us consider

$$
L(x):=\sup \left\{\alpha \in \mathbb{R}: x-\alpha e_{1} \in G\right\}, x \in \mathbb{R}^{D} .
$$

In finance, $L(x)$ is the liquidation value of the financial position $x$. As $-e_{1} \notin K$, we may show that $L(x)$ is a maximum, i.e. $x-L(x) e_{1} \in G$ for all $x \in \mathbb{R}^{d}$. In particular, $G=\left\{x \in \mathbb{R}^{d}: L(x) \geqslant 0\right\}$.

Let us define the random preorder $x \gg y$ if $x-y \in G$. By the assumptions on $G$, this is a random order and $x \gg y$ if and only if $L(x-y) \geqslant 0$. As $G+G \subset G$, we obtain that $L(x+y) \geqslant L(x)+L(y)$, i.e. $L$ is super additive.

Let us show that $L$ is upper semi-continuous (u.s.c.). Consider a convergent sequence $\left(x_{n}\right)_{n \in \mathbb{N}}$ whose limit is $x$. Recall that $x_{n}-L\left(x_{n}\right) e_{1} \in$ $G$. In the case where $\left|L\left(x_{n}\right)\right| \rightarrow+\infty$ for a subsequence, then using the normalization $\tilde{x}_{n}:=x_{n} /\left(1+\left|L\left(x_{n}\right)\right|\right) \rightarrow 0$, we get that $-e_{1} \in K$ as $n \rightarrow \infty$ hence a contradiction. We deduce that $\lim _{\sup _{n}} L\left(x_{n}\right)<\infty$ and, as $n \rightarrow \infty, x-\lim \sup _{n} L\left(x_{n}\right) e_{1} \in G$. This implies that $L(x) \geqslant$ $\lim \sup _{n} L\left(x_{n}\right)$. At last, $(\omega, x) \mapsto L(\omega, x)$ is $\mathcal{F} \times \mathcal{B}\left(\mathbb{R}^{d}\right)$-measurable. Indeed, as $\mathbb{R}_{+} e_{1} \subset G$, we may show that, for every $c \in \mathbb{R},\{(\omega, x) \in$ $\left.\Omega \times \mathbb{R}^{d}: L(\omega, x) \geqslant c\right\}=\left\{(\omega, x) \in \Omega \times \mathbb{R}^{d}: x-c e_{1} \in G\right\}$, the latter set belonging to $\mathcal{F} \times \mathcal{B}\left(\mathbb{R}^{d}\right)$ since $G$ is measurable.

Finally, we suppose that the points of the boundary $\partial G$ may be separated by a bounded $\mathcal{F}$-normal integrand $u$ in the sense that $x-y \in$ $\partial G$ implies that $u(x)>u(y)$ is $x \neq y$. In that case, $\gg$ is of type I since $\{L=0\} \subset \partial G$. This condition will be satisfied in the financial model we shall present below.

Example 3.5. Let $G$ be a random $\mathcal{F}$-measurable closed convex cone of $\mathbb{R}^{d}, d \geqslant 1$, which contains 0 . The positive dual is $G^{*}:=\left\{x \in \mathbb{R}^{d}: x g \geqslant\right.$ $0, \forall g \in G\}$. It is well know that $G=\left(G^{*}\right)^{*}$ and $G^{*}$ is $\mathcal{F}$-measurable. Therefore, $G^{*}$ admits a Castaing representation $G^{*}=\operatorname{cl}\left(g_{i}^{*}: i \in \mathbb{N}\right\}$ and $G=\left\{x \in \mathbb{R}^{d}: x g_{i}^{*} \geqslant 0, \forall i \in \mathbb{N}\right\}$. Therefore, if we define $x \gg y$ by $x-y \in G$, we get that $x \gg y$ if and only if $u_{i}(x) \geqslant u_{i}(y)$ a.s. for every $i \in \mathbb{N}$, where $u_{i}(z)=g_{i}^{*} z$.

The following concepts are introduced to solve minimization problems related to random preorders. See also [10], [11] and [12] where similar notions are considered. 
Lemma 3.6. Let $X \subset L^{0}\left(\mathbb{R}^{d}, \mathcal{F}\right)$ be such that $X_{\mathcal{H}}:=X \cap L^{0}\left(\mathbb{R}^{d}, \mathcal{H}\right) \neq$ $\varnothing$. Suppose that $\gg$ is antisymmetric, i.e. $\gg$ is a random order. There exists a unique subset $\hat{X} \subset X_{\mathcal{H}}$ denoted by $\hat{X}=\operatorname{Essmin}_{\mathcal{H}}(X)$ satisfying the following properties:

(i) For all $\gamma \in X_{\mathcal{H}}$ :, there exists $\hat{\gamma} \in \hat{X}$ such that $\gamma \gg \hat{\gamma}$.

(ii) If $\hat{\gamma}_{1}, \hat{\gamma}_{2} \in \hat{X}$ satisfy $\hat{\gamma}_{1} \gg \hat{\gamma}_{2}$, then $\hat{\gamma}_{1}=\hat{\gamma}_{2}$.

Proof. Suppose that there exists two subsets $\hat{X}_{1}, \hat{X}_{2} \subset X_{\mathcal{H}}$ satisfying (i) and (ii). Consider any $\hat{\gamma}_{1} \in \hat{X}_{1}$. As $\hat{X}_{2}$ satisfies (i), there exists $\hat{\gamma}_{2} \in \hat{X}_{2}$ such that $\hat{\gamma}_{1} \gg \hat{\gamma}_{2}$. Similarly, there exists $\tilde{\gamma}_{1} \in \hat{X}_{1}$ such that $\hat{\gamma}_{2} \gg \tilde{\gamma}_{1}$. We deduce by transitivity that $\hat{\gamma}_{1} \gg \tilde{\gamma}_{1}$. This implies by (ii) that $\hat{\gamma}_{1}=\tilde{\gamma}_{1}$. Therefore, $\hat{\gamma}_{1} \geqslant \hat{\gamma}_{2}$ and $\hat{\gamma}_{2} \geqslant \hat{\gamma}_{1}$. By antisymmetry, we get that $\hat{\gamma}_{1}=\hat{\gamma}_{2} \in \hat{X}_{2}$, i.e. $\hat{X}_{1} \subset \hat{X}_{2}$. By symmetry, we also get that $\hat{X}_{2} \subset \hat{X}_{1}$ so that the equality holds.

We may only consider random preorders if we only consider a weak version of the essential minimum. To do so, we write $\gamma_{1} \sim \gamma_{2}$ if $\gamma_{1} \gg \gamma_{2}$ and $\gamma_{2} \gg \gamma_{1}$.

Lemma 3.7. Let $X \subset L^{0}\left(\mathbb{R}^{d}, \mathcal{F}\right)$ be such that $X_{\mathcal{H}}:=X \cap L^{0}\left(\mathbb{R}^{d}, \mathcal{H}\right) \neq$ $\varnothing$. There exists a unique subset $\hat{X} \subset X_{\mathcal{H}}$ denoted by $\hat{X}=\operatorname{Essmin}_{\mathcal{H}}^{w}(X)$ satisfying the following properties:

(i) For all $\gamma \in X_{\mathcal{H}}$, there exists $\hat{\gamma} \in \hat{X}$ such that $\gamma \gg \hat{\gamma}$.

(ii) If $\hat{\gamma}_{1}, \hat{\gamma}_{2} \in \hat{X}$ satisfy $\hat{\gamma}_{1} \gg \hat{\gamma}_{2}$, then $\hat{\gamma}_{1} \sim \hat{\gamma}_{2}$.

(iii) For any $\hat{\gamma} \in \hat{X}, \tilde{\gamma} \sim \hat{\gamma}$ and $\tilde{\gamma} \in X_{\mathcal{H}}$, implies $\tilde{\gamma} \in \hat{X}$.

Proof. It suffices to repeat the proof of Lemma 3.6 where we use (iii) instead of the antisymmetry condition.

When necessary, we denote by $\operatorname{Essmin}_{\mathcal{H}}^{\gg}(X)$ or $\operatorname{Essmin}_{\mathcal{H}}^{w, »}(X)$ the essential minimum $\operatorname{Essmin}_{\mathcal{H}}(X)$ or $\operatorname{Essmin}_{\mathcal{H}}^{w}(X)$ to specify the chosen preorder.

Example 3.8 (Minimization on a random set). Let $D$ be a random $\mathcal{F}$ measurable closed set we consider as the domain of control variables $x \in \mathbb{R}^{d}$ satisfying some random contraints. Let $c$ be a random cost function we suppose to be a $\mathcal{F}$-normal integrand. Our goal is to study the problem of minimising $c$ over $D$. Notice that this problem is a priori considered pointwise, i.e. for each $\omega \in \Omega$, we may solve the problem $\min _{x \in D(\omega)} c(\omega, x)$. Here, we seek for mesurable minimizers of the problem with values in $D$. This is why we introduce the following random preorder of type II: $x \gg y$ if $c(x) \gg c(y)$ a.s. Actually, we may show that $\min _{x \in D(\omega)} c(\omega, x)=c(\hat{\gamma})$ a.s. whatever $\hat{\gamma} \in \operatorname{Essmin}_{\mathcal{F}}^{w}\left(L^{0}(D, \mathcal{F})\right) \neq \varnothing$, see Theorem 6.1. 


\section{MAin RESUlts}

The goal of this section is to provide conditions under which the essential minimum $\hat{X}=\operatorname{Essmin}_{\mathcal{H}}(X)\left(\right.$ resp. $\left.\operatorname{Essmin}_{\mathcal{H}}^{w}(X)\right)$ of some family of random variables $X \subset L^{0}\left(\mathbb{R}^{d}, \mathcal{H}\right)$ is not empty.

Theorem 4.1. Let $X \subset L^{0}\left(\mathbb{R}^{d}, \mathcal{H}\right)$. Suppose that $X_{\mathcal{H}}=X \cap$ $L^{0}\left(\mathbb{R}^{d}, \mathcal{H}\right) \neq \varnothing$ is $\mathcal{H}$-decomposable and closed in $L^{0}$. Assume that for any $\gamma \in X_{\mathcal{H}}$ and any sequence $\left(\gamma_{n}\right)_{n \in \mathbb{N}} \in X_{\mathcal{H}}$ such that $\gamma_{n} \ll \gamma$, we have $\lim \inf _{n}\left|\gamma_{n}\right|<\infty$ a.s. Suppose that the random preorder 》 is of type $I$ (resp. of type II). Then, $\hat{X}=\operatorname{Essmin}_{\mathcal{H}}(X) \neq \varnothing$ (resp. $\left.\operatorname{Essmin}_{\mathcal{H}}^{w}(X) \neq \varnothing\right)$.

Proof. Suppose that $\gg$ is of type I, i.e. represented by a function $L$ in Definition 3.2 such that $x \gg y$ if and only if $L(x-y) \geqslant 0$. Let us define $\hat{L}(z):=\arctan L(z) \in[-\pi / 2 ; \pi / 2]$. For any $\gamma \in X_{\mathcal{H}}$, we denote by $L^{0}((-\infty, \gamma], \mathcal{H})$ the set of all $\mathcal{H}$-measurable random variables $\tilde{\gamma}$ such that $\tilde{\gamma} \ll \gamma$ and we define

$$
a(\gamma)=\inf _{\left.\tilde{\gamma} \in X_{\mathcal{H}} \cap L^{0}((-\infty, \gamma]), \mathcal{H}\right)} \mathrm{E} \hat{L}(\gamma-\tilde{\gamma})
$$

By definition, there exists a sequence $\left(\gamma_{n}\right)_{n \in \mathbb{N}} \in X_{\mathcal{H}} \cap L^{0}((-\infty, \gamma], \mathcal{H})$ such that $a(\gamma)=\lim _{n} \mathrm{E} \hat{L}\left(\gamma-\gamma_{n}\right)$.

Let us consider the sequence $\left(\tilde{\gamma}_{n}\right)_{n \in \mathbb{N}}$ defined by $\tilde{\gamma}_{1}=\gamma_{1}$ and its next terms are recursively defined as

$$
\tilde{\gamma}_{n+1}=\gamma_{n+1} 1_{\mathrm{E}\left(\hat{L}\left(\gamma-\gamma_{n+1}\right) \mid \mathcal{H}\right)>\mathrm{E}\left(\hat{L}\left(\gamma-\tilde{\gamma}_{n}\right) \mid \mathcal{H}\right)}+\tilde{\gamma}_{n} 1_{\mathrm{E}\left(\hat{L}\left(\gamma-\gamma_{n+1}\right) \mid \mathcal{H}\right) \leqslant \mathrm{E}\left(\hat{L}\left(\gamma-\tilde{\gamma}_{n}\right) \mid \mathcal{H}\right)} .
$$

Since $X_{\mathcal{H}}$ is $\mathcal{H}$-decomposable, we deduce by induction that $\left(\tilde{\gamma}_{n}\right)_{n \in \mathbb{N}} \in$ $X_{\mathcal{H}} \cap L^{0}((-\infty, \gamma], \mathcal{H})$. Moreover, by construction, the sequence $\mathrm{E}(\hat{L}(\gamma-$ $\left.\left.\tilde{\gamma}_{n+1}\right) \mid \mathcal{H}\right)$ is non decreasing and satisfies $\mathrm{E}\left(\hat{L}\left(\gamma-\tilde{\gamma}_{n+1}\right) \mid \mathcal{H}\right) \geqslant \mathrm{E}(\hat{L}(\gamma-$ $\left.\left.\gamma_{n+1}\right) \mid \mathcal{H}\right)$ hence $a(\gamma)=\lim _{n} \mathrm{E} \hat{L}\left(\gamma-\tilde{\gamma}_{n}\right)$.

By the assumption, $\lim \inf _{n}\left|\tilde{\gamma}_{n}\right|<\infty$ a.s. Therefore, by [13, Lemma 2.1.2], there exists a $\mathcal{H}$-measurable sequence $n_{k} \in L^{0}(\mathbb{N}, \mathcal{H})$ such that $\tilde{\gamma}_{n_{k}}$ is a.s. convergent to some $\gamma_{\infty} \in L^{0}\left(\mathbb{R}^{d}, \mathcal{H}\right)$. As $X_{\mathcal{H}}$ is $\mathcal{H}$ decomposable and closed, we deduce that

$$
\tilde{\gamma}_{n_{k}}=\sum_{j \geqslant k} \tilde{\gamma}_{j} 1_{n_{k}=j} \in X_{\mathcal{H}} \cap L^{0}((-\infty, \gamma], \mathcal{H})
$$

It follows that $\gamma_{\infty} \in X_{\mathcal{H}}$. Moreover, since $\hat{L}$ is u.s.c., $\hat{L}\left(\gamma-\gamma_{\infty}\right) \geqslant$ $\lim \sup _{k} \hat{L}\left(\gamma-\tilde{\gamma}_{n_{k}}\right) \geqslant 0$ hence $\gamma_{\infty} \ll \gamma$. At last,

$$
\mathrm{E}\left(\hat{L}\left(\gamma-\tilde{\gamma}_{n_{k}}\right) \mid \mathcal{H}\right)=\sum_{j \geqslant k} \mathrm{E}\left(\hat{L}\left(\gamma-\tilde{\gamma}_{j}\right) \mid \mathcal{H}\right) 1_{n_{k}=j} \geqslant \mathrm{E}\left(\hat{L}\left(\gamma-\tilde{\gamma}_{k}\right) \mid \mathcal{H}\right)
$$


Therefore, $a(\gamma)=\lim _{k} \mathrm{E}\left(\hat{L}\left(\gamma-\tilde{\gamma}_{n_{k}}\right)\right)$ and, since $\hat{L}$ is u.s.c.,

$$
\begin{gathered}
a(\gamma) \geqslant \mathrm{E}\left(\hat{L}\left(\gamma-\gamma_{\infty}\right)\right) \geqslant \mathrm{E}\left(\limsup _{k} \hat{L}\left(\gamma-\tilde{\gamma}_{n_{k}}\right)\right) \\
\quad \underset{k}{\limsup } \mathrm{E}\left(\hat{L}\left(\gamma-\tilde{\gamma}_{n_{k}}\right)\right)=a(\gamma) .
\end{gathered}
$$

It follows that $a(\gamma)=\mathrm{E}\left(\hat{L}\left(\gamma-\gamma_{\infty}\right)\right)$ is a minimum. Let us define

$$
\Lambda(\gamma):=\left\{\gamma_{\infty} \in X_{\mathcal{H}} \cap L^{0}((-\infty, \gamma], \mathcal{H}): a(\gamma)=\mathrm{E}\left(\hat{L}\left(\gamma-\gamma_{\infty}\right)\right)\right\} \neq \varnothing
$$

For any $\gamma_{\infty} \in \Lambda(\gamma)$, consider $\tilde{\gamma} \in X_{\mathcal{H}} \cap L^{0}((-\infty, \gamma], \mathcal{H})$ such that $\tilde{\gamma} \ll \gamma_{\infty}$. As $L$ is super additive, $L(\gamma-\tilde{\gamma}) \geqslant L\left(\gamma-\gamma_{\infty}\right)+L\left(\gamma_{\infty}-\tilde{\gamma}\right)$ where $L\left(\gamma_{\infty}-\tilde{\gamma}\right) \geqslant 0$ by assumption. Therefore, $L(\gamma-\tilde{\gamma}) \geqslant L\left(\gamma-\gamma_{\infty}\right)$ and the inequality is strict on $L\left(\gamma_{\infty}-\tilde{\gamma}\right)>0$. This implies that $a(\gamma) \geqslant$ $\mathrm{E}(\hat{L}(\gamma-\tilde{\gamma}))>\mathrm{E}\left(\hat{L}\left(\gamma-\gamma_{\infty}\right)\right)$ if $\mathrm{P}\left(L\left(\gamma_{\infty}-\tilde{\gamma}\right)>0\right)>0$. This contradicts the definition of $a(\gamma)$ hence $L\left(\gamma_{\infty}-\tilde{\gamma}\right)=0$. For any $\gamma_{\infty} \in \Lambda(\gamma)$, we then define

$$
b\left(\gamma_{\infty}\right):=\inf _{\tilde{\gamma} \in X_{\mathcal{H}} \cap L^{0}\left(\left(-\infty, \gamma_{\infty}\right], \mathcal{H}\right)} \mathrm{E} u(\tilde{\gamma}),
$$

where $u$ is given in Definition 3.2. As above, we may show that $b\left(\gamma_{\infty}\right)=$ $\mathrm{E} u(\hat{\gamma})$ for some $\hat{\gamma} \in X_{\mathcal{H}} \cap L^{0}\left(\left(-\infty, \gamma_{\infty}\right], \mathcal{H}\right)$. Let us define $\hat{\Lambda}\left(\gamma_{\infty}\right):=\{\hat{\gamma} \in$ $\left.X_{\mathcal{H}} \cap L^{0}\left(\left(-\infty, \gamma_{\infty}\right], \mathcal{H}\right)\right\} \neq \varnothing$ and

$$
\hat{X}:=\bigcup_{\gamma \in X_{\mathcal{H}}} \bigcup_{\gamma_{\infty} \in \Lambda(\gamma)} \hat{\Lambda}\left(\gamma_{\infty}\right) .
$$

Let us verify that $\hat{X}$ satisfies the conditions of Lemma 3.6. First $\hat{X} \subset$ $X_{\mathcal{H}}$ and $\hat{X} \neq \varnothing$ by construction since $X_{\mathcal{H}} \neq \varnothing$. Let us consider $\gamma \in \hat{X}$. Then, $\gamma \gg \gamma_{\infty}$ for $\gamma_{\infty} \in \Lambda(\gamma) \neq \varnothing$ and $\gamma_{\infty} \gg \hat{\gamma}$ where $\hat{\gamma} \in \hat{\Lambda}\left(\gamma_{\infty}\right) \neq \varnothing$. It follows that Condition (i) holds.

Suppose that $\hat{\gamma}^{1} \gg \hat{\gamma}^{2}$ where $\hat{\gamma}^{1}, \hat{\gamma}^{2} \in \hat{X}$. We have $\hat{\gamma}^{1} \in \hat{\Lambda}\left(\gamma_{\infty}^{1}\right)$ where $\gamma_{\infty}^{1} \in \Lambda\left(\gamma^{1}\right)$ for some $\gamma^{1} \in X_{\mathcal{H}}$ such that $\hat{\gamma}^{1} \ll \gamma_{\infty}^{1} \ll \gamma^{1}$. As above, we deduce that $L\left(\gamma_{\infty}^{1}-\hat{\gamma}^{1}\right)=L\left(\gamma_{\infty}^{1}-\hat{\gamma}^{2}\right)=0$. As $L$ is super additive, we also have $L\left(\gamma-\hat{\gamma}^{2}\right) \geqslant L\left(\gamma-\gamma_{\infty}^{1}\right)+L\left(\gamma_{\infty}^{1}-\gamma_{\infty}^{2}\right)$. We then deduce as above that $L\left(\gamma_{\infty}^{1}-\gamma_{\infty}^{2}\right)=0$ since $a\left(\gamma^{1}\right)=\mathrm{E} L\left(\gamma-\gamma_{\infty}^{1}\right)$. On the set where $\hat{\gamma}^{1} \neq \hat{\gamma}^{2}$, we deduce that $u\left(\gamma_{\infty}^{1}\right)>u\left(\gamma_{\infty}^{2}\right)$ hence $b\left(\gamma_{\infty}^{1}\right)=\mathrm{E} u\left(\hat{\gamma}^{1}\right)>$ $\mathrm{E} u\left(\hat{\gamma}^{2}\right)$ if $\mathrm{P}\left(\hat{\gamma}^{1} \neq \hat{\gamma}^{2}\right)>0$ in contradiction with the definition of $b\left(\gamma_{\infty}^{1}\right)$. Therefore, $\hat{\gamma}^{1}=\hat{\gamma}^{2}$ and Condition (ii) holds.

Suppose that $\gg$ is of type II, i.e. $\gg$ is represented by a countable family of random l.s.c. functions $\left(u_{i}\right)_{i \in \mathbb{N}}$ in Definition 3.3 such that $x \gg y$ if and only if $u_{i}(x) \geqslant u_{i}(y)$ for all $i \in \mathbb{N}$. We may assume without loss of generality that $\left|u_{i}\right|$ is bounded by 1 for every $i \in \mathbb{N}$. Indeed, 
replace $u_{i}$ by $\frac{2}{\pi} \arctan \left(u_{i}\right)$. We then define $u(x)=\sum_{i=1}^{\infty} 2^{-1} u_{i}(x)$. We may show that $u$ is l.s.c. For any $\gamma \in X_{\mathcal{H}}$, we consider

$$
a(\gamma)=\inf _{\left.\tilde{\gamma} \in X_{\mathcal{H}} \cap L^{0}((-\infty, \gamma]), \mathcal{H}\right)} \mathrm{E} u(\tilde{\gamma}) .
$$

As in the first proof above, we get $a(\gamma)=\mathrm{E}\left(u\left(\gamma_{\infty}\right)\right)$ for some $\gamma_{\infty} \in$ $X_{\mathcal{H}} \cap L^{0}((-\infty, \gamma], \mathcal{H})$. We define

$$
\Lambda(\gamma):=\left\{\gamma_{\infty} \in X_{\mathcal{H}} \cap L^{0}((-\infty, \gamma], \mathcal{H}): a(\gamma)=\mathrm{E}\left(u\left(\gamma_{\infty}\right)\right)\right\} \neq \varnothing
$$

and we claim that

$$
\operatorname{Essmin}_{\mathcal{H}}^{w}(X)=\bigcup_{\gamma \in X_{\mathcal{H}}} \Lambda(\gamma) \neq \varnothing
$$

Indeed, Condition (i) of Definition 3.7 holds by construction. Condition (iii) is trivially satisfied as $\tilde{\gamma} \sim \gamma$ implies that $u(\tilde{\gamma})=u(\gamma)$. At last, consider $\gamma^{1}, \gamma^{2} \in \operatorname{Essmin}_{\mathcal{H}}^{w}(X)$ such that $\hat{\gamma}^{1} \gg \hat{\gamma}^{2}$. We have $\hat{\gamma}^{1} \in \Lambda\left(\gamma^{1}\right)$ for some $\gamma^{1} \in X_{\mathcal{H}}$ such that $a\left(\gamma^{1}\right)=\mathrm{E}\left(u\left(\hat{\gamma}^{1}\right)\right)$. Then, $\gamma^{1} \gg \hat{\gamma}^{1} \gg \gamma^{2}$ implies that $\mathrm{E}\left(u\left(\hat{\gamma}^{1}\right)\right) \geqslant \mathrm{E}\left(u\left(\hat{\gamma}^{2}\right)\right) \geqslant a\left(\gamma^{1}\right)$. We deduce that $\mathrm{E}\left(u\left(\hat{\gamma}^{1}\right)-\right.$ $\left.u\left(\hat{\gamma}^{2}\right)\right)=0$ hence $u\left(\hat{\gamma}^{1}\right)-u\left(\hat{\gamma}^{2}\right)=0$ and finally $u_{i}\left(\hat{\gamma}^{1}\right)-u_{i}\left(\hat{\gamma}^{2}\right)=0$ for all $i$, i.e. $\hat{\gamma}^{1} \sim \hat{\gamma}^{2}$.

\section{Application in Finance}

On a complete stochastic basis $\left(\Omega,\left(\mathcal{F}_{t}\right)_{t=0, \cdots, T}, \mathrm{P}\right)$, we consider a financial market model defined by a sequence of random sets $\left(G_{t}\right)_{t=0, \cdots, T}$ such that $G_{t}$ is $\mathcal{F}_{t}$-measurable at any instant $t=0, \cdots, T$ where $T \geqslant 1$ is an horizon date. We suppose that, for any $t, G_{t}$ satisfies the conditions of Example 3.4. In particular, the liquidation value process is given by

$$
L_{t}(x):=\sup \left\{\alpha \in \mathbb{R}: x-\alpha e_{1} \in G\right\}, x \in \mathbb{R}^{D}
$$

so that $G_{t}=\left\{x: L_{t}(x) \geqslant 0\right\}$ is the set of all solvent financial portfolio positions which can be liquidated without any debt. In the following, we define the associated random orders $\gg_{t}$ by $x \gg_{t} y$ if and only if $x-y \in G_{t}$. Notice that $\gg_{t}$ is of type I.

A classical 2-dimensional model is to consider a Bond $S_{t}^{0}=1$ (w.l.o.g.) and a risky asset defined by Bid and Ask prices $S^{b}$ and $S^{a}$ such that $S^{b} \leqslant S^{a}$. Recall that $S^{b}$ is the price you get when selling one unit of risky asset and $S^{a}$ is the price you pay when buying one unit of risky asset. In that case, $L_{t}((x, y))=x+y^{+} S_{t}^{b}-y^{-} S_{t}^{a}$ where $x^{+}:=\max (x, 0)$ and $x^{-}=-\min (x, 0)$. Moreover, $G_{t}$ is a convex cone containing $\mathbb{R}_{+}^{2}$ whose positive dual is the smallest convex cone containing $\{1\} \times\left[S_{t}^{b}, S_{t}^{a}\right]$. In [15], there is also a model where a fixed

cost is charged for every transaction. In that case, the solvency sets 
$\left(G_{t}\right)_{t=0, \cdots, T}$ are no more convex but still define an order satisfying the conditions of Example 3.4.

Definition 5.1. A portfolio process is a stochastic process $\left(V_{t}\right)_{t=0, \cdots, T}$ adapted to $\left(\mathcal{F}_{t}\right)_{t=0, \cdots, T}$, i.e. $V_{t} \in L^{0}\left(\mathbb{R}^{d}, \mathcal{F}_{t}\right)$ for all $t=0, \cdots, T$, such that

$$
\Delta V_{t}:=V_{t}-V_{t-1} \in-G_{t}, \quad t=0, \cdots, T .
$$

The interpretation of the dynamics (5.1) is simple: we may write $V_{t-1}=V_{t}+\left(-\Delta V_{t}\right)$ so that it is possible to change $V_{t-1}$ into $V_{t}$ since we may liquidate the residual term $\left(-\Delta V_{t}\right)$ without any debt. We may also interpret (5.1) as the paiement of transaction costs when changing $V_{t-1}$ into $V_{t}$, see [13, Section 3]. Notice that (5.1) may be reformulated as $V_{t-1} \gg_{t} V_{t}$ for all $t \leqslant T$.

The classical problem in finance is to characterise the set of all portfolio processes whose terminal values are larger that some given contingent claim $\xi \in L^{0}\left(\mathbb{R}^{d}, \mathcal{F}_{T}\right)$ of a financial derivative, i.e. some wealth $\xi$ which is delivered at the maturity date upon paiement at time 0 . The mathematical problem is then to estimate the prices of $\xi$ at any time $t$, i.e. the values $V_{t}$ of portfolio processes super hedging $\xi$, i.e. $V_{T} \geqslant \xi$.

In general, in particular for models without transaction costs, a no arbitrage condition is imposed to characterise the prices of a contingent claim $\xi$, see [13, Section 2]. In the following, we suppose the no arbitrage condition called (NA2) ${ }^{2}$, initially introduced by M. Ràsonyi [17]. In [15], it is proven that (NA2) is equivalent to $L^{0}\left(G_{t+1}, \mathcal{F}_{t}\right) \subset L^{0}\left(G_{t}, \mathcal{F}_{t}\right)$ for all $t \leqslant T-1$. We may reformulate this equivalent condition as $\mathrm{m}\left(G_{t+1} \mid \mathcal{F}_{t}\right) \subset G_{t}$ a.s. for all $t \leqslant T-1$, where $\mathrm{m}\left(G_{t+1} \mid \mathcal{F}_{t}\right)$ is the largest $\mathcal{F}_{t}$-measurable set contained in $G_{t+1}$, called conditional core, see [14]. Under (NA2), we may characterise the minimal portfolio processes super hedging $\xi$ as follows. Let us denote by $\mathcal{H}^{\xi}$ the set of all portfolio processes super hedging $\xi$.

Definition 5.2. A portfolio process $\left(\hat{V}_{t}\right)_{t=0, \cdots, T}$ of $\mathcal{H}^{\xi}$ is said minimal if whatever $V \in \mathcal{H}^{\xi}$, the condition $\hat{V}_{t} \gg_{t} V_{t}$ implies that $\hat{V}=V$.

Notice that $\hat{V}_{t} \gg_{t} V_{t}$ means that $\hat{V}_{t}=V_{t}+g_{t}$ where $g_{t} \in L^{0}\left(G_{t}, \mathcal{F}_{t}\right)$, i.e. we need to add the extra position $g_{t}$ to $V_{t}$ to obtain $\hat{V}_{t}$. If we introduce the minimal cost function $C_{t}(z):=\inf \left\{\alpha \in \mathbb{R}: \alpha e_{1}-z \in G_{t}\right\}$, i.e. the minimal amount of cash we need to get the position $z$, then $C_{t}(z)=-L_{t}(-z)$. Therefore, $C_{t}\left(g_{t}\right)=-L_{t}\left(-g_{t}\right)$ where $L_{t}\left(-g_{t}\right) \geqslant 0$ if and only if $-g_{t} \in G_{t} \cap\left(-G_{t}\right)=\{0\}$. Therefore, if $g_{t} \neq 0$, then $C_{t}\left(g_{t}\right)>0$ so that it is expensive to change $V_{t}$ into $\hat{V}_{t}$. This motivates Definition

\footnotetext{
${ }^{2}$ Absence of arbitrage of second kind.
} 
5.2. The natural problem is about the existence of minimal portfolio processes and a characterisation of them. We denote by $\mathcal{H}_{\text {min }}^{\xi}$ the set of all minimal portfolio processes super hedging $\xi$ and by $\mathcal{H}_{\text {min }}^{\xi}(t)$ their values at time $t$. The following result improves [15, Proposition 4.9], which is only formulated for the random preorder defined by $G_{t+1}$ instead of $G_{t}$ here, by providing a backward characterization of $\mathcal{H}_{\text {min }}^{\xi}$.

Theorem 5.3. Suppose that Condition (NA2) holds. Let $\xi \in$ $L^{0}\left(\mathbb{R}^{d}, \mathcal{F}_{T}\right)$ be such that $\mathcal{H}^{\xi} \neq \varnothing$. Then, $\mathcal{H}_{\text {min }}^{\xi} \neq \varnothing$ and $\left(\mathcal{H}_{\text {min }}^{\xi}(t)\right)_{t=0, \cdots, T}$ is characterized recursively by $\mathcal{H}_{\text {min }}^{\xi}(T)=\xi$ and $\mathcal{H}_{\text {min }}^{\xi}(t)=\operatorname{Essmin}_{\mathcal{F}_{t}}^{\gg_{t}}\left(\mathcal{H}_{\text {min }}^{\xi}(t+1)+L^{0}\left(G_{t+1}, \mathcal{F}_{t+1}\right)\right) \neq \varnothing, t \leqslant T-1$

Proof. It is clear that $\mathcal{H}_{\text {min }}^{\xi}(T)=\xi$. Let us consider $V \in \mathcal{H}^{\xi} \neq \varnothing$. Then, $V_{T-1} \in X_{\mathcal{F}_{T-1}}=X_{T} \cap L^{0}\left(\mathbb{R}^{d}, \mathcal{F}_{T-1}\right)$ where $X_{T}=L^{0}\left(G_{T}, \mathcal{F}_{T}\right)+$ $\xi$. Notice that $X_{\mathcal{F}_{T-1}}$ is closed in $L^{0}$. Let us now verify that, for any $\gamma \in L^{0}\left(\mathbb{R}^{d}, \mathcal{F}_{T-1}\right)$ and for all sequence $\left(\gamma_{n}\right)_{n \in \mathbb{N}}$ of $X_{\mathcal{F}_{T-1}}$ such that $\gamma_{n} \ll_{T-1} \gamma$, we have $\liminf _{n}\left|\gamma_{n}\right|<\infty$ a.s. To see it, observe that $\xi \ll_{T} \gamma_{n} \ll_{T-1}$. On the $\mathcal{F}_{T-1}$ measurable set $F_{T-1}=\left\{\liminf _{n}\left|\gamma_{n}\right|=\right.$ $\infty\}$, divide the previous inequality by $1+\left|\gamma_{n}\right|$ and we may assume by $\left[13\right.$, Lemma] that $\tilde{\gamma}_{n}:=\gamma_{n} /\left(1+\left|\gamma_{n}\right|\right) \rightarrow \tilde{\gamma}$ on $F_{T-1}$ such that $|\tilde{\gamma}|=1$ and $0 \ll_{T} \tilde{\gamma} \ll_{T-1} 0$. In particular, $\tilde{\gamma} \in L^{0}\left(G_{T}, \mathcal{F}_{T-1}\right)$ hence, under (NA2), $\tilde{\gamma} \in \mathrm{m}\left(G_{T} \mid \mathcal{F}_{T-1}\right) \subset G_{T-1}$. This implies that $\tilde{\gamma} \in G_{T-1} \cap$ $\left(-G_{T-1}\right)=\{0\}$ in contradiction with $|\tilde{\gamma}|=1$. Therefore, $\mathrm{P}\left(F_{T-1}\right)=0$ hence $\liminf \operatorname{in}_{n}\left|\gamma_{n}\right|<\infty$ a.s.

By Theorem 4.1, we set $\mathcal{H}_{\text {min }}^{\xi}(T-1)=\operatorname{Essmin}_{\mathcal{F}_{T-1}}^{\gg_{T-1}}\left(L^{0}\left(G_{T}, \mathcal{F}_{T}\right)+\xi\right) \neq$ $\varnothing$. If $V \in \mathcal{H}^{\xi} \neq \varnothing$, then $V_{T-2} \gg_{T-1} V_{T-1}$ where $V_{T-1} \in X_{\mathcal{F}_{T-1}}$. This implies that $V_{T-1} \gg_{T-1} \hat{V}_{T-1}$ for some $\hat{V}_{T-1} \in \mathcal{H}_{\text {min }}^{\xi}(T-1)$. Therefore, $V_{T-2} \in X_{\mathcal{F}_{T-2}}=X_{T-1} \cap L^{0}\left(\mathbb{R}^{d}, \mathcal{F}_{T-2}\right)$ where $X_{T-1}=\mathcal{H}_{\text {min }}^{\xi}(T-1)+$ $L^{0}\left(G_{T-1}, \mathcal{F}_{T-1}\right)$. By the previous reasoning with Theorem 4.1, we then set $\mathcal{H}_{\text {min }}^{\xi}(T-2)=\operatorname{Essmin}_{\mathcal{H}}^{\gg_{T-2}}\left(\mathcal{H}_{\text {min }}^{\xi}(T-1)+L^{0}\left(G_{T-1}, \mathcal{F}_{T-1}\right)+\right) \neq$ $\varnothing$. We then reiterate the construction. Starting from $\mathcal{H}_{\text {min }}^{\xi}(T)=$ $\xi$, it is then possible to backwardly generate a minimal portfolio, i.e. from $\hat{V}_{t+1} \in \mathcal{H}_{\text {min }}^{\xi}(t+1)$, it suffices to consider $\hat{V}_{t} \in$ $\operatorname{Essmin}_{\mathcal{F}_{t}}^{\gg_{t}}\left(\hat{V}_{t+1}+L^{0}\left(G_{T-1}, \mathcal{F}_{T-1}\right)+\right) \neq \varnothing$. Reciprocally, by construction, any $V_{0} \in \mathcal{H}_{\text {min }}^{\xi}(0)$ is the initial value of some minimal portfolio process $\hat{V}$ with $\hat{V}_{t} \in \mathcal{H}_{\text {min }}^{\xi}(t)$ for all $t \leqslant T$. Moreover, it is clear by construction that, for any $V \in \mathcal{H}^{\xi}$, there exists $\hat{V} \in \mathcal{H}_{\text {min }}^{\xi}$ such that $V_{t} \gg_{t} \hat{V}_{t}$ for all $t \leqslant T$, which also proves that the sets $\mathcal{H}_{\text {min }}^{\xi}(t), t \leqslant T$, generate $\mathcal{H}_{\text {min }}^{\xi} \neq \varnothing$. 


\section{RANDOM MINIMISATION ON A RANDOM SET}

In this section, we develop Example 3.8. As announced in the example, we are interested in $\operatorname{Essmin}_{\mathcal{F}}^{w}(X)$ where $X=L^{0}(D, \mathcal{F})$ is the set of all measurable controls. Indeed, in the case where it is not empty, then any $\gamma \in L^{0}(D, \mathcal{F})$ may be reduced into a cheaper control $\hat{\gamma} \in \operatorname{Essmin}_{\mathcal{F}}^{w}(X) \subset L^{0}(D, \mathcal{F})$, i.e. such that $c(\gamma) \geqslant c(\hat{\gamma})$. We then claim that any $\hat{\gamma} \in \operatorname{Essmin}_{\mathcal{F}}^{w}(X) \subset L^{0}(D, \mathcal{F})$ satisfies $\min _{x \in D(\omega)} c(\omega, x)=$ $c(\hat{\gamma})$.

Theorem 6.1. Consider a random $\mathcal{F}$-measurable set $D$. Let $c$ be a (random) $\mathcal{F}$-normal integrand and consider the random order $x \gg y$ if $c(x) \geqslant c(y)$. Suppose that the associated essential minimum (see Definition 3.7) $\operatorname{Essmin}_{\mathcal{F}}^{w}\left(L^{0}(D, \mathcal{F})\right)$ is not empty. Then,

$$
\min _{x \in D(\omega)} c(\omega, x)=c(\hat{\gamma}), \text { a.s. }
$$

whatever $\hat{\gamma} \in \operatorname{Essmin}_{\mathcal{F}}^{w}\left(L^{0}(D, \mathcal{F})\right)$.

To prove this result, we need to recall the notion of real-valued essential infinimum of a family of random variables, see [13, Section 5.3]:

Proposition 6.2. Let $\left(\gamma_{i}\right)_{i \in I}$ be a family of $\mathcal{F}$-measurable random variables with values in $[-\infty, \infty]$ on a complete probability space $(\Omega, \mathcal{F}, \mathrm{P})$. There exists a unique (up to a negligible set) $\mathcal{F}$-measurable random variable $\bar{\gamma} \in L^{0}([-\infty, \infty], \mathcal{F})$ such that:

(i) $\bar{\gamma} \leqslant \gamma_{i}$ a.s. for every $i \in I$.

(ii) If $\gamma \in L^{0}([-\infty, \infty], \mathcal{F})$ satisfies $\gamma \leqslant \gamma_{i}$ a.s. for every $i \in I$, then $\gamma \leqslant \bar{\gamma}$ a.s.

Proof of Theorem 6.1.

We first prove that for all $\hat{\gamma}_{1}, \hat{\gamma}_{2} \in \operatorname{Essmin}_{\mathcal{F}}^{w}(X), c\left(\hat{\gamma}_{1}\right)=c\left(\hat{\gamma}_{2}\right)$, i.e. $\hat{\gamma}_{1} \sim \hat{\gamma}_{2}$. To see it, consider $\hat{\gamma}=\hat{\gamma}_{1} 1_{c\left(\hat{\gamma}_{1}\right)>c\left(\hat{\gamma}_{2}\right)}+\hat{\gamma}_{2} 1_{c\left(\hat{\gamma}_{2}\right) \geqslant\left(\hat{\gamma}_{2}\right)}$. Then, $\hat{\gamma} \ll \hat{\gamma}^{i}, i=1,2$, hence $\hat{\gamma} \in \operatorname{Essmin}_{\mathcal{F}}^{w}(X)$ and $\hat{\gamma} \sim \hat{\gamma}^{1} \sim \hat{\gamma}^{2}$.

Secondly, we show that $\operatorname{essinf}_{\mathcal{F}}\{c(\gamma): \gamma \in X\}=c(\hat{\gamma})$ whatever $\hat{\gamma} \in \operatorname{Essmin}_{\mathcal{F}}^{w}(X)$. Indeed, $\left.\operatorname{ess}_{\inf }\{c(\gamma): \gamma \in X\}\right) \leqslant c(\hat{\gamma})$ by definition of the essential supremum. On the other hand, for all $\gamma \in X$, there is $\hat{\gamma} \in \operatorname{Essmin}_{\mathcal{F}}^{w}(X)$ such that $\gamma \gg \hat{\gamma}$ hence the conclusion follows.

Finally, let us consider that the $\mathcal{F}$-measurable set $F(\omega):=\{x \in$ $D(\omega): c(\omega, x)<c(\omega, \hat{\gamma}(\omega)\}$ where $\hat{\gamma}$ is chosen arbitrarily in $\operatorname{Essmin}_{\mathcal{F}}^{w}(X)$. By a measurable selection argument, there exists on the set $\{\omega: F(\omega) \neq \varnothing\}$ a measurable selection $\tilde{\gamma} \in L^{0}(F, D)$. Then, $\bar{\gamma}=\tilde{\gamma} 1_{F \neq \varnothing}+\hat{\gamma} 1_{F=\varnothing} \in L^{0}(D, \mathcal{F})$ satisfies $\bar{\gamma} \leqslant \hat{\gamma}$. As $\hat{\gamma} \in \operatorname{Essmin}_{\mathcal{F}}^{w}(X)$, we deduce that $\bar{\gamma} \sim \hat{\gamma}$ hence $P(F \neq \varnothing)=0$. It follows that a.s. for 
all $x \in D, c(\omega, x) \geqslant c(\hat{\gamma})$ hence $\min _{x \in D(\omega)} c(\omega, x)=c(\hat{\gamma})$, which also proves that $\min _{x \in D(\omega)} c(\omega, x)$ is $\mathcal{F}$-measurable. In conclusion, for all $\hat{\gamma} \in \operatorname{Essmin}_{\mathcal{F}}^{w}\left(L^{0}(D, \mathcal{F})\right)$,

$$
\min _{x \in D(\omega)} c(\omega, x)=\operatorname{essinf}_{\mathcal{F}}\left\{c(\gamma): \gamma \in L^{0}(D, \mathcal{F})\right\}=c(\hat{\gamma})
$$

i.e. the pointwise minimization of the random function $c$ on the random set $D$ is solved by identifying the elements of $\operatorname{Essmin}_{\mathcal{F}}^{w}(X)$.

Remark 6.3. By Theorem 4.1, $\operatorname{Essmin}_{\mathcal{F}}^{w}\left(L^{0}(D, \mathcal{F})\right) \neq \varnothing$ when $D$ is a.s. compact. This is also the case when $D$ is closed and the (real-valued) cost function satisfies $\lim _{|z| \rightarrow \infty} c(z)=+\infty$. Indeed, in that case, if $\left(\gamma_{n}\right)_{n \in \mathbb{N}}$ satisfies $\gamma_{n} \ll \gamma$, i.e. $c\left(\gamma_{n}\right) \leqslant c(\gamma)$ for some $\gamma \in L^{0}\left(\mathbb{R}^{d}, \mathcal{F}\right)$, then on the set $\left\{\liminf _{n}\left|\gamma_{n}\right|=+\infty\right\}$, we obtain $\infty \leqslant c(\gamma)$, i.e. a contradiction so that $\liminf _{n}\left|\gamma_{n}\right|<+\infty$.

\section{REFERENCES}

[1] Baptiste J., Carassus L. and Lépinette E. Pricing without martingale measures. Preprint. https://hal.archives-ouvertes.fr/hal-01774150.

[2] Lohne A. and Rudloff B. An algorithm for calculating the set of superhedging portfolios in markets with transaction costs.Int. J. Theor. Appl. Finan. 17, 2014 (02): S. 1450012-[33 p].

[3] Dalang E.C., Morton A. and W. Willinger. Equivalent martingale measures and no-arbitrage in stochastic securities market models. Stochastics and Stochastic Reports, 1990, 29, 185-201.

[4] C. Hess. Set-valued integration and set-valued probability theory: An overview. In E. Pap, editor, Handbook of Measure Theory, chapter 14, pages 617-673. Elsevier, 2002.

[5] Hiai F. and Umegaki H. Integrals, conditional expectations, and martingales of multivalued functions. J. Multiv. Anal., 7:149-182, 1977.

[6] Feinstein Z. and Rudloff B. Multi-portfolio time consistency for set-valued convex and coherent risk measures. Finance and Stochastics 19 (1), 2015, S. $67-107$.

[7] Hamel A., Heyde F. and Rudloff B. Set-valued risk measures for conical market models. Math Finan Econ 5 (1), 2011, S. 1-28.

[8] Jamneshan A., Kupper M. and Zapata J. M. Parameter-dependent Stochastic Optimal Control in Finite Discrete Time. Preprint. https://arxiv.org/ abs/1705.02374v1.

[9] Jouini E. and Kallal H. Martingales and arbitrage in securities markets with transaction costs. Journal of Economic Theory, 66(1), 1995, 178-1997.

[10] Kabanov Y. and Lépinette E. Essential supremum with respect to a random partial order.Journal of Mathematical Economics, 49 (2013), 6, 478-487.

[11] Kabanov Y. and Lépinette E. Essential supremum and essential maximum with respect to random preference relations. Journal of Mathematical Economics, 49 (2013), 6, 488-495. 
[12] Kabanov Y. and Lépinette E. On supremal and maximal sets with respect to random partial orders. "Set Optimization - State of the Art and Applications in Finance." Ed. A. Hamel, Springer, 151(2015), 275-291.

[13] Kabanov Y. and Safarian M. Markets with transaction costs. Mathematical Theory. Springer-Verlag, 2009.

[14] Lépinette E. and IMolchanov I.. Conditional cores and conditional convex hulls of random sets. Preprint, submitted. https://arxiv.org/abs/1605. 07884

[15] Lépinette E. and Tran T. General financial market model defined by a liquidation value process. Stochastics, 88 (2016), 3, 437-459.

[16] Molchanov I. Theory of Random Sets. Springer, London, 2005.

[17] Ràsonyi M. Arbitrage Under Transaction Costs Revisited. Optimality and Risk-Modern Trends in Mathematical Finance, (2009), 211-225. Springer, Berlin, Heidelberg.

[18] Rockafellar R. T. and Wets R. J.-B.. Variational analysis, volume 317 of Grundlehren der Mathematischen Wissenschaften [Fundamental Principles of Mathematical Sciences]. Springer-Verlag, Berlin, 1998. ISBN 3-540-62772-3.

Ceremade, Paris-Dauphine University, Place du Maréchal De Lattre De Tassigny, 75775 Paris Cedex 16, France, and, Gosaef, Faculté des Sciences de Tunis, Manar II-Tunis, Tunisia.

E-mail address: emmanuel.lepinette@ceremade.dauphine.fr 